\title{
Induced Disruption in the Connectivity of an Identified Neuron in the Drosophila ts Mutant shibire
}

\author{
Margaret Raper Hummon and Walter J. Costello \\ Department of Zoological and Biomedical Sciences/College of Osteopathic Medicine, Ohio University, Athens, Ohio \\ 45701
}

Temperature-sensitive mutants permit the selective expression of mutant genotype. The Drosophila ts mutant shibire (shi) is paralytic at $30^{\circ} \mathrm{C}$; the probable primary effect of the mutation is disruption of membrane recycling. In studying the development of the giant fiber (GF) pathway during the pupal period, we find that shi flies exposed to heat pulse during early pupal states exhibit perturbation in the development of an identified neuron that links giant fibers to motoneurons of indirect flight muscles. Concomitantly, latency in activation of these muscles by the giant fiber pathway is significantly increased. Flies exposed to heat pulse during the late pupal period remain similar to control shi and wildtype flies in giant fiber pathway anatomy and muscle latency. Thus, the critical period of development of an identified neuron in a known motor pathway can be defined by its period of sensitivity to the shi defect. The time-dependent defect is apparently specific for cells that are at a developmental stage that is greatly dependent on membrane recycling processes. Use of this mutant will allow us to investigate the possible role of membrane recycling in development and to establish critical periods of neuronal development.

A developing neuron must grow and form connections with specific end targets to construct a mature nervous system. In embryonic systems, mechanisms involved in pathfinding to the end target appear to include differential adhesion, surface recognition factors, guidepost cells, and pioneer fibers (Goodman and Pearson, 1982). How these mechanisms influence the neuron in finding the correct pathway is still unknown.

Holometabolous insects such as Drosophila melanogaster exist in 3 forms during their life cycle: as larva, as pupa, and as adult. Metamorphosis from larva to adult occurs during the pupal stage. In this process, the pupa of Drosophila retains many elements of the larval nervous system, as well as developing complex neural networks to control uniquely adult structures, such as compound eyes, articulated legs, wings, and genitalia. During the prepupal period, the larval muscular system commences degeneration; subsequently, adult muscles develop. Neurons serving adult structures must solve developmental challenges similar to those of the embryo, i.e., to find correct

\footnotetext{
Received Dec. 9, 1986; revised May 7, 1987; accepted May 8, 1987.

We thank R. A. DiCaprio, R. S. Hikida, E. H. Peterson, and R. J. Wyman for helpful comments on the manuscript, and Y.-T. Kim and C.-F. Wu for providing a preprint of their paper. This work was supported in part by grants from Ohio University Research Committee (W.J.C.), Muscular Dystrophy Association of America (W.J.C.), and research funds from the Ohio University College of Osteopathic Medicine (W.J.C.).

Correspondence should be addressed to M. R. Hummon at the above address. Copyright (C) 1987 Society for Neuroscience $0270-6474 / 87 / 113633-06 \$ 02.00 / 0$
}

pathways both within and outside the central nervous system and to establish appropriate synapses with targets.

To study the factors involved in nerve connectivity, we are investigating the development of a simple motor system, the giant fiber (GF) pathway, during pupal development in D. melanogaster. The adult GF pathway develops during the pupal period and mediates the escape response, which consists of a jump and subsequent flight. The adult GF pathway is bilateral, with a pair of GFs passing from the brain through the cervical connective and into the thoracic ganglion (Fig. 1). At the midthoracic neuromere, each GF makes morphological contact, first with a peripherally synapsing interneuron (PSI), and then with the motoneuron to the tergotrochanteral, or "jump," muscle (TTM) (King and Wyman, 1980). The PSI sends its axon out a peripheral nerve (PDMN), where PSI makes chemical synapses onto 5 motor axons that synapse with the wing depressors (DLM). On the basis of the minimum latency and synchrony of excitatory postsynaptic potentials in target muscles following brain stimulation, the morphological contacts in the ganglion (GF to PSI and to the TTM axon, PSI to PSI) are thought to contain electrical synapses (Tanouye and Wyman, 1980).

Drosophila carrying the temperature-sensitive mutation shibire (shi) are heat-sensitive paralytics, and fail to survive when reared continuously at nonpermissive temperatures $\left(30^{\circ} \mathrm{C}\right)$. However, shi flies reared at the permissive temperature $\left(22^{\circ} \mathrm{C}\right)$ resemble wild-type flies in behavior, appearance, and survival (Poodry et al., 1973), and have a similar pattern of TTM and DLM response after GF stimulation (Thomas and Wyman, 1984). Both larvae and adults undergo rapid paralysis at $30^{\circ} \mathrm{C}$ and rapid recovery at $22^{\circ} \mathrm{C}$ (Grigliatti et al., 1973; Poodry et al., 1973). Paralysis in the adult is preceded by an increased spontaneous activity of nerves and muscles (Salkoff and Kelly, 1978) and is associated with morphological depletion of synaptic vesicles at synapses (Poodry and Edgar, 1979; Koenig et al., 1983; Kosaka and Ikeda, 1983a) and with the accumulation of coated pits at synapses and in actively endocytotic cells (Kosaka and Ikeda, 1983a, b).

Exposure to heat pulse $\left(6 \mathrm{hr}, 30^{\circ} \mathrm{C}\right)$ during early pupal development can result in several anomalies in the adult; e.g., partial fusion of ommatidia (Poodry et al., 1973) and of leg segments (Costello and Salkoff, 1983), patterned changes in the number of micro- and macrochaetes (Poodry et al., 1973; Hummon and Costello, 1986a), reduction in the number of fibers in the wing depressor muscles (Costello and Salkoff, 1983), and presence of neuromas in adults with partial deletion of target muscles (Hummon and Costello, 1986a). The temperature-sensitive and rapidly reversible paralysis in adults is apparently 


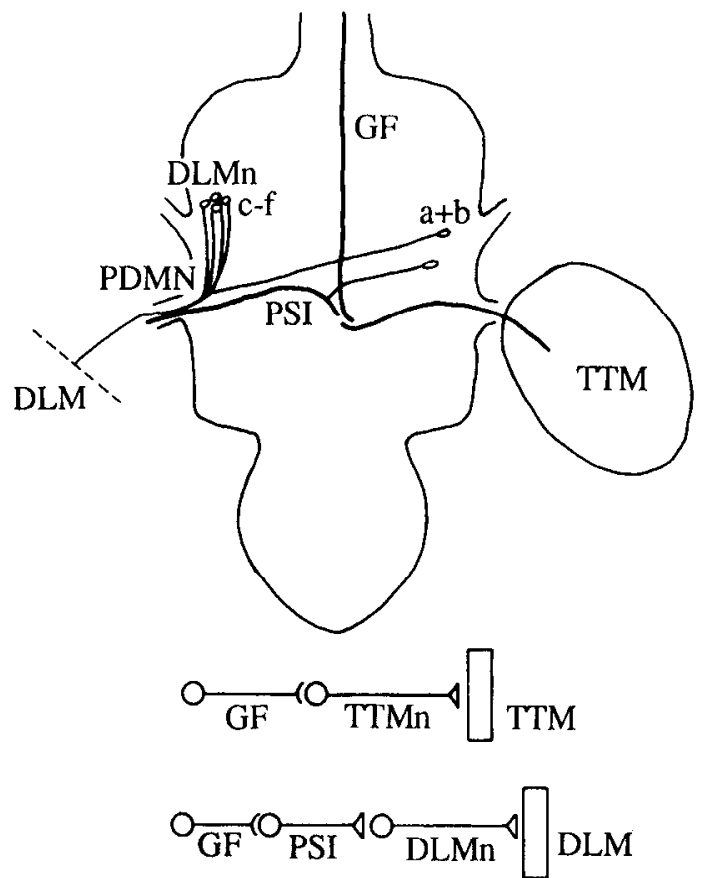

Figure 1. The GFP in the thoracic ganglion of adult Drosophila melanogaster in wild-type or temperature-sensitive mutant shi flies reared at the permissive temperature $\left(22^{\circ} \mathrm{C}\right)$. In the ganglion, $a-f$ refer to cell bodies of the 5 DLMns. Electrical synapses between GF and PSI, GF and TTMn; chemical synapses between PSI and DLMn, DLMn and DLM, TTMn and TTM. Adapted in part from Coggshall (1978) and King and Wyman (1980).

related to defects in membrane constituents that are detrimental only at elevated temperatures. Nonpermissive temperatures also affect shi neurons in culture, causing reduced rates of neurite branching and elongation and a lack of lamellipodia in growth cones (Kim and $\mathrm{Wu}, 1986,1987)$. A common chracteristic of both developmental and functional anomalies appears to be the involvement of endocytotic but not exocytotic mechanisms during development (Koenig et al., 1983; Costello and Salkoff, 1986), although the biochemical basis of the defect is as yet unknown and more complex explanations are possible (Kim and $\mathrm{Wu}$, 1987).

We report that by using the temperature-sensitive mutation $s h i$, with its effect on membrane recycling (Kosaka and Ikeda, 1983a, b; Costello and Hummon, 1985; Costello and Salkoff, 1986), we can perturb aspects of pupal neurogenesis by exposure of pupae to heat pulse. Specific changes in anatomy and function are determined by the stage of pupal development at the time of onset of heat pulse (Costello and Salkoff, 1983; Hummon and Costello, 1985, 1986a). We report here that one neuronal element of the GF pathway can be selectively disrupted in the Drosophila mutant shi, with correlated alterations in electrophysiology and anatomy. Such a time-dependent and cell-specific alteration allows us to define critical periods of pathway devclopment in identified neurons.

\section{Materials and Methods}

Wild-type flies (Canton-S) and stock with the allele $s h i^{i s}$ were maintained at 18 or $22^{\circ} \mathrm{C}$. Wandering third-stage larvae were removed to moist filter paper in petri dishes and held at room temperature $(22$ $24^{\circ} \mathrm{C}$ ) until the formation of white prepupae (considered time 0 ; total pupal period at $25^{\circ} \mathrm{C}$ lasts $96 \mathrm{hr}$ ). To facilitate comparison, all pupal ages have been converted to the $25^{\circ} \mathrm{C}$ equivalent (no. of hr at $22^{\circ} \mathrm{C} \times$ $0.67=$ no. of $\mathrm{hr}$ at $25^{\circ} \mathrm{C}$ ). For heat pulse experiments, shi pupae of known ages were kept at $22^{\circ} \mathrm{C}$ throughout the pupal period, except for a heat pulse of $6 \mathrm{hr}\left(30^{\circ} \mathrm{C}\right)$ at specific times during pupal development.

Extracellular stimulation of the GF in adults permitted us to identify the latency of activation of the TTM and DLM (Tanouye and Wyman, 1980; Thomas and Wyman, 1984). GF stimulation at low voltage may initiate GF pathway activation with longer and more variable latencies; at higher voltage, the minimum-latency GF pathway is activated (Tanouye and Wyman, 1980). All latencies reported here are the shortest times that could be elicited from suprathreshold stimulation $($ at $0.2 \mathrm{~Hz}$ ) of GFs, which effectively eliminates long-latency GF pathway activation. Adults were 1-3 d old when tested. Previously reported mean minimum latencies $(\mathrm{msec})$ for wild-type flies (Canton-S) include 0.81 for TTM and 1.25 for DLM (Tanouye and Wyman, 1980), as well as 0.88 for TTM and 1.30 for DLM (Thomas and Wyman, 1984). Differences in latency probably reflect differences in recording temperatures; at $22^{\circ} \mathrm{C}$ we find 0.88 for TTM, and 1.31 for DLM (Table 1 ).

In a series of heat pulse experiments, shi flies were subjected to heat pulse with onset at a series of short intervals throughout the pupal period (at 12, 16, 20, 24, 32, 44, 60, and $78 \mathrm{hr} ; n=4-10$ flies for each group), or with heat pulse omitted (control). Major differences were not observed between short intervals of heat pulse onset, except where noted in the text. Thus, we have used major increments to bracket developmental periods (onset at $12,20,44,60$, and $78 \mathrm{hr}$ ). All recordings were at $22^{\circ} \mathrm{C}$ with temperature controlled with a Cambion Bipolar controller and temperature stage. Minimum latencies were statistically compared using Wilcoxon's nonparametric 2 -sample test for significant differences $(\alpha-$ 0.05) (Sokal and Rohlf, 1969), with control shi as reference for each treatment group. In both wild-type and control shiflies, we have recently found inherent differences in latency between DLM fibers $a / b$ and fibers c-f following GF stimulation (M. R. Hummon and W. J. Costello,

Table 1. Effect of pupal heat pulse (HP) $\left(6 \mathrm{hr}, 30^{\circ} \mathrm{C}\right)$ on minimum latency of escape response muscles in shi adults $\left(22^{\circ} \mathrm{C}\right)$

\begin{tabular}{|c|c|c|c|c|c|c|c|c|c|}
\hline & & \multicolumn{4}{|l|}{ TTM } & \multicolumn{4}{|l|}{ DLM } \\
\hline & $\begin{array}{l}\text { HP onset } \\
(\mathrm{hr})\end{array}$ & $N(n)$ & $\begin{array}{l}\bar{x} \\
\text { (msec) }\end{array}$ & $s^{2}$ & $p$ & $N(n)$ & $\begin{array}{l}\bar{x} \\
\text { (msec) }\end{array}$ & $s^{2}$ & $p$ \\
\hline Canton $S$ & None & $10(19)$ & 0.88 & 0.003 & & $10(20)$ & 1.31 & 0.021 & \\
\hline$s h i$ & None & $10(20)$ & 0.88 & 0.002 & & $10(19)$ & 1.39 & 0.009 & \\
\hline shi & 12 & $5 \quad(9)$ & 0.93 & 0.011 & ns & 5 (9) & 1.56 & 0.033 & $*$ \\
\hline$s h i$ & 44 & $8(16)$ & 0.97 & 0.015 & $*$ & $8(15)$ & 1.64 & 0.028 & $* *$ \\
\hline$s h i$ & 78 & $3(6)$ & 0.87 & 0.002 & ns & $3(6)$ & 1.38 & 0.011 & ns \\
\hline
\end{tabular}

Formation of white prepupa is at $0 \mathrm{hr}$; subsequent hours converted to $25^{\circ} \mathrm{C}$ (see text). Nonparametric Wilcoxon 2-sample test for significant differences between $s h i$ following heat pulse and $s h i$ controls (no heat pulse). For comparison, minimum latencies are also given for wild-type (Canton S) flies reared at $22^{\circ} \mathrm{C}$ (no significant difference from shi controls).

HP, heat pulse; $N$. number of adults used for recordings; $n$, number of measurements; $\bar{x}$, mean; $s^{2}$, variance. $p$. significance: ${ }^{*} p>0.05 ;{ }^{* *} p>0.001 ;$ ns, not significant. 

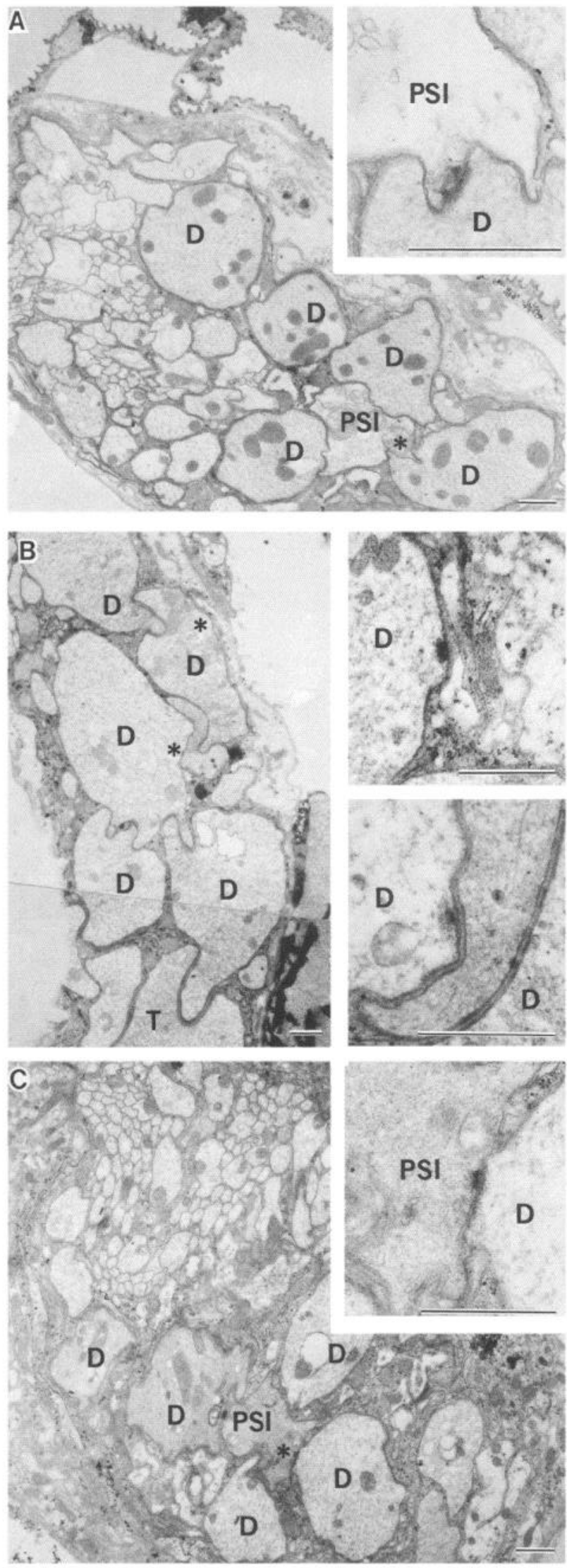

Figure 2. Cross sections of PDMN near divergence (10-20 $\mu \mathrm{m})$ from mesothoracic ganglion; nerve and axons traced serially with light microscopy and TEM. $A$, shi reared at $22^{\circ} \mathrm{C}$ throughout pupal develop- 1986b, and unpublished observations). Thus, all latency values for DLM (Table 1) are for fibers c-f only.

Each fly was tested electrophysiologically to determine latency of DLM and TTM activation from GF stimulation. Following the recording session, flies were fixed and prepared for light and electron microscopy (TEM). Epon-embedded thoraces were serially thick-sectioned (5 $\mu \mathrm{m})$ and examined under glycerin-mounted coverslips by phase microscopy. Intact thoraces and selected thick sections re-embedded for TEM (King et al., 1982) were serially thin-sectioned and examined with a Zeiss 109 TEM. A minimum of 2 flies (equal 4 PDMNs) was examined from each heat pulse onset time $(12,20,44,60$, and $78 \mathrm{hr}$ of pupal development) or with no heat pulse.

\section{Results}

\section{Survival}

Survival of control $\left(22^{\circ} \mathrm{C}\right)$ and experimental $\left(22^{\circ} \mathrm{C}\right.$ except $6 \mathrm{hr}$ pupal heat pulse) shi flies resembles that reported previously (Poodry et al., 1973). Controls show about $95 \%$ unaided emergence $(n=164)$. Early pupal heat pulse prior to head eversion (onset $<9 \mathrm{hr}$ ) is lethal, while onset of heat pulse at $12-78 \mathrm{hr}$ allows full development in most flies $(80 \% ; n=397)$, with eclosion maximally impaired (50-100\%) for onset of heat pulse at 10-24 hr of development.

Failure of interneuron PSI to reach normal target area in the peripheral nerve PDMN after early pupal heat pulse

Control shi flies never subjected to heat pulse have a normal GF pathway. The GFs are present, following the same course as in wild-type flies. The PSI extends from the midthoracic neuromere of the ganglion $40-50 \mu \mathrm{m}$ into a peripheral nerve, the PDMN (Fig. 2A). Within the PDMN, PSI forms frequent synapses onto axons of the DLM motor neurons and receives occasional synapses from the DLM motor axons (not shown). The TTM motor axon is usually within the PDMN for a distance of about $10-45 \mu \mathrm{m}$, and then diverges to the TTM; occasionally the TTM motor axon has a separate origin from the thoracic ganglion.

In flies with heat pulse from early (12 or $20 \mathrm{hr}$ ) or mid- (44 or $60 \mathrm{hr}$ ) pupal stages, most components of the GF pathway appear morphologically normal. The GFs are present; the PDMN includes the axons of the 5 DLM motoneurons and the axon of the TTM motoneuron. However, the PSI never occurs in its normal locale in flies subjected to heat pulse during early pupal stages (onset at 12 or $20 \mathrm{hr} ; 7$ PDMNs) (Fig. 2 B). Only in the base of the PDMN is the axonal profile of PSI present; it extends at most for about $10 \mu \mathrm{m}$ into the PDMN from the thoracic ganglion. In this abnormal terminus area, the PSI forms some chemical synapses onto the DLM motor axons and occasionally receives multiple synapses from the DLM motor axons. In the distal part of PDMN, where the PSI terminus normally occurs, the DLM motor axons form abnormal synapses, usually onto space or glia, or sometimes adjacent to another axon; such con-

ment; TTMn present only in more proximal PDMN. Inset, Chemical synapse in nearby section, from PSI to DLMn. $B$, shi reared at $22^{\circ} \mathrm{C}$ except for $6 \mathrm{hr} \mathrm{HP}, 20 \mathrm{hr}$ onset; TTMn present. Insets, hemisynapses in adjacent section; in DLMn facing glial cell (upper) and in DLMn facing small axon (lower). C, shi reared at $22^{\circ} \mathrm{C}$ except for $6 \mathrm{hr}$ pupal heat pulse, $78 \mathrm{hr}$ onset. On this side, TTMn had a separate origin from the ganglion and was never within the PDMN. Inset, Chemical synapse in adjacent section, from PSI to DLMn. In $A$ and $C$. PSI is prominent at this level; in $B$, the PSI is absent. $D$, DLMn; $T$, TTMn. Scale bar, 1 $\mu \mathrm{m}$. 
A

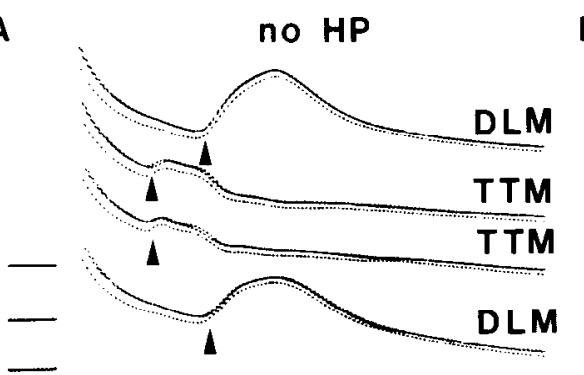

B

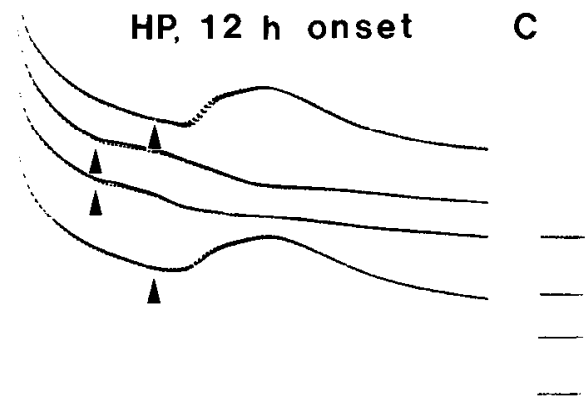

HP, 78 h onset

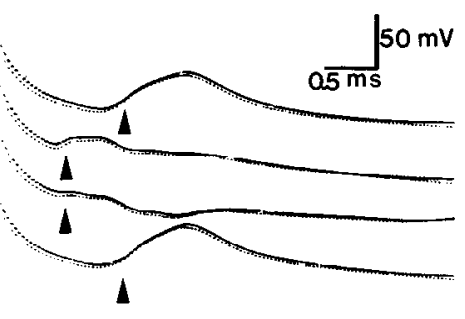

Figure 3. Electrophysiology of GFP of adult shi; recordings from right and left target muscles (TTM, middle traces; DLM, upper and lower traces) at $22^{\circ} \mathrm{C}$. Arrowheads indicate the mean latency for shi flies without HP. $A$, shi reared at $22^{\circ} \mathrm{C}$ throughout pupal develpment. $B, s h i$ reared at $22^{\circ} \mathrm{C}$ except for $6 \mathrm{hr}$ pupal heat pulse, $12 \mathrm{hr}$ onset. $C$, shi reared at $22^{\circ} \mathrm{C}$ except for $6 \mathrm{hr}$ pupal heat pulse, $78 \mathrm{hr}$ onset.

ditions are rarely seen $(<5 \%$ of sections examined) in control flies or in flies subjected to late pupal heat pulse (onset at 78 hr). These abnormal synapses resemble "hemisynapses," having presynaptic thickenings and vesicles, but lacking any postsynaptic specialization (Fig. $2 B$, inset).

In flies receiving heat pulse late in pupal development $(78 \mathrm{hr})$, the components of GF pathway are normal. The morphology and locale of PSI are similar to those of control flies (Fig. 2C). However, when flies receive the heat pulse about $24 \mathrm{hr}$ earlier (44 or $60 \mathrm{hr}$ onset), the morphology of the PSI in PDMN becomes highly variable. In almost half the PDMNs examined (5 PDMNs), the PSI was virtually normal, being prominent in the thoracic ganglion, extending well into the PDMN to its normal locale, and synapsing onto the axons of the DLM motoneurons. In the remainder (6 PDMNs), PSI was either completely absent from PDMN or occurred only at its base, where it resembled in structure and synaptic populations those adults that were exposed to heat pulse during early pupal development. Hemisynapses in DLM axons were also present in the PDMN where PSI would normally occur, resembling the condition seen in flies subjected to early pupal heat pulse. In terms of overall morphology and connection to target muscle, the DLM motor axons and the TTM motor axon remained normal regardless of heat pulse timing.

\section{Increased latency of $G F$ pathway muscle (DLM) response in flies subjected to early or midpupal heat pulse}

Wild-type and shi fiies reared at $22^{\circ} \mathrm{C}$ resemble each other in the GF pathway latencies of TTM and DLM activation. There are no statistically significant differences between wild-type and control shi flies (Table 1).

In adult shi flies exposed to heat pulse as pupae in early (12 $\mathrm{hr}$ ) or mid- ( $44 \mathrm{hr}$ ) pupal stages, the DLM latency is significantly higher than in control groups (Table 1, Fig. 3). In flies subjected to heat pulse late in the pupal period $(78 \mathrm{hr})$, the minimum latency for DLM is similar to that of the control shi flies.

In contrast, the minimum latency for TTM is only minimally affected by heat pulse during early and midpupal stages, with means not consistently different from controls' (Table 1, Fig. 3). Additional studies on TTM activation are in progress to determine if the slight latency differences are consistent and to investigate the GF-TTM axon contacts in the thoracic ganglion.

\section{Discussion}

Metamorphosis in Drosophila is from a crawling to a walking and flying animal, with drastic alteration in shape, musculature, and innervation. The pupal nervous system undergoes considerable reorganization. Both pathfinding and synaptogenesis must occur in several elements within a particular time frame to produce the adult GF pathway. As demonstrated here, heat pulse in shi has an effect on DLM latency and PSI development only during particular periods of pupal development. In contrast, the routine presence of DLM and TTM motor axons and their neuromuscular responses following GF pathway activation indicates that DLM and TTM motor axons are not disrupted by pupal heat pulse, regardless of time. The insensitivity of the motoneurons innervating DLM and TTM to pupal heat pulse is understandable if these motoneurons are indeed modified larval motoneurons (Costello and Thomas, 1981; Costello, 1986), and hence are in their target area prior to pupation. These motoneurons would thus not require extensive growth and pathfinding to reach their end targets.

The PSI, however, is affected by heat pulse according to the time of onset. The PSI during pupal development must contact the GF within the thoracic ganglion, where dual electrical and chemical synapses are formed; as well, the PSI must extend out the PDMN some distance to form chemical synapses with DLM motor axons in a specific area (King and Wyman, 1980). Heat pulse during early pupal stages ( 12 or $20 \mathrm{hr}$ ) always affects the PSI. Heat pulse late in pupal development $(78 \mathrm{hr})$ never affects the PSI. Yet heat pulse at intermediate periods (44 or $60 \mathrm{hr}$ ) has variable effects on the anatomy of PSI; in some cases PSI is normal, and in others it is abnormal. This suggests that the intermediate period is the approximate endpoint of PSI growth. The variability is likely due to minor differences in developmental timing, with cells in some flies connecting more quickly than in others.

There must also be critical interactions between PSI and DLM motor axons during this sensitive period, since heat pulse always causes significant disruption in electrophysiology. However, even when PSI is abnormal, responses occur with GF stimulation, albeit with a significant incrcase in DLM latency. The PSI plays an intergral role in the escape response. Consistent and complete activation of the 5 DLM motor axons is a requisite for such a role. Therefore, it is likely that the PSI in a normal GF pathway has a large safety factor in its synaptic output to ensure DLM motor axon activation. Hence, the increase in DLM latency is most likely due to the altered morphology of PSI. Such altered morphology includes (1) the absence of PSI beyond the base of PDMN, which causes (2) the aberrant locale of PSI synapses onto DLM motor axons, and (3) the altered structure and number of those synapses extant. 
However, by $78 \mathrm{hr}$ of pupal development, heat pulse no longer disrupts PSI development; as well, DLM latency from GF stimulation resembles controls. This nonsensitive period for PSI coincides closely with the time ( $>70 \mathrm{hr}$ ) at which the entire GF pathway can be activated in wild-type pupae (Costello and Thomas, 1981; W. J. Costello, unpublished observations). Hence, the reason for the lack of effect on PSI by heat pulse after the intermediate period is most likely that PSI has already completed its growth and established synaptic connections.

Another mutation of Drosophila, gfA, exhibits even longer DLM latency (mean, $3.21 \mathrm{msec}$ ) (Thomas and Wyman, 1984). It is interesting that the presumed effect of $g f A$, resulting in longer latency, might also involve an alteration in PSI, either in GF to PSI connection or in PSI synapses onto DLM motor axons. As yet, neither developmental events nor the morphological or functional substrates of this phenotype have been determined.

In the normal PDMN, PSI makes frequent chemical synapses onto DLM motor axons; reciprocal synapses between PSI and DLM motor axons also occur (see also King and Wyman, 1980). The widespread occurrence of hemisynapses made by DLM motor axons in the aberrant PDMNs is surprising. In the usual terminus zone of PSI, though PSI is absent, DLM motor axons contain numerous presynaptic specializations, including synaptic vesicles, which abut onto space, glia, or axons lacking postsynaptic specialization (Fig. $2 B$ ). Such a condition rarely occurs in flies having a PDMN containing a normal PSI. The fact that DLM motor axons maintain and apparently accumulate presynaptic structures suggests that the absence of a postsynaptic structure has not prevented DLM motor axon synaptogenesis, normally occurring in this locale. Hemisynapses have also been observed in muscle-deletion mutants of Drosophila (Costello and Wyman, 1986), in neuromas (Hirano and Shin, 1979), and in tissue of flightless grasshoppers persisting after programmed muscle loss (Arbas and Tolbert, 1986). The common factor in all these cases is an aberrant postsynaptic cell. The hemisynapses differ from the "presynaptic tables" of interneurons facing glia in the first visual ganglion of the fly Musca (Burkhardt and Braitenberg, 1976), the latter being much larger and associated with intercellular and postsynaptic glial cell specializations.

Of interest is the lack of catastrophic perturbation to the development and survival of shi pupae exposed to heat pulse after $10 \mathrm{hr}$ of pupal development. Presumably all membrane recycling is interrupted during the heat pulse. Processes such as endocytosis (Kosaka and Ikeda, 1983a, b; Costello and Salkoff, 1986) or maintenance of growth cone structures (Kim and Wu, 1986, 1987) are impaired. Yet most pupal structures develop. This pattern implies that membrane recycling mechanisms are widespread and critical during the very early pupal period $(<10$ hr after formation of white prepupa), since exposure of shi pupae to heat pulse at that time results in complete lethality. Thereafter, heat pulse has a more limited effect, such that overall development continues and only specific anomalies occur. This would imply that membrane recycling mechanisms specifically involved in development are then more limited, occurring at high levels in only select tissues during restricted times.

The actual membrane recycling mechanisms crucial to proper formation of the PSI remain unknown. However, because of the shi defect, the processes involved in membrane recycling, e.g., endocytosis, growth cone formation, or a combination of both, are certainly implicated. For instance, in normal systems growing nerve fibers apparently induce endocytosis (coated vesicle formation) in specific cells contacted during pathway formation (Bastiani and Goodman, 1984). In shi pupae fixed just after a heat pulse with onset at $13 \mathrm{hr}$, we observe an accumulation of endocytotic vesicles in remnants of larval muscles and in the surrounding adult myocytes at the site of DLM development (Costello and Hummon, 1985). This situation is never observed in older pupae, containing more mature DLM. As well, in cultures of shi neurons, neurite growth is greatly suppressed at restrictive temperatures that disrupt growth cone structure (Kim and $\mathrm{Wu}, 1986,1987)$.

Hence, the most significant variable determining a structure's proper formation is time of onset of heat pulse (see also Poodry ct al., 1973). Thus the critical period of PSI sensitivity to heat pulse reported here probably corresponds to PSIs peak development, when membrane recycling mechanisms may play a significant role in the formation of proper connectivity. One such mechanism, endocytosis, is certainly implicated, according to the evidence that the shi defect causes a disruption in the endocytotic process (Poodry and Edgar, 1979; Kosaka and Ikeda, 1983a, b; Costello and Hummon, 1985; Costello and Salkof, 1986). Such results provide evidence that further links membrane recycling processes with proper pathfinding by growing nerve fibers or migrating cells (Bastiani and Goodman, 1984; Costello and Hummon, 1985; Kim and Wu, 1986, 1987). In addition, they permit us to establish critical periods for the development of selected components within a motor system.

\section{References}

Arbas, E. A., and L. P. Tolbert (1986) Presynaptic terminals persist following degeneration of "flight" muscle during development of a flightless grasshopper. J. Neurobiol. 17: 627-636.

Bastiani, M. J., and C. S. Goodman (1984) Neuronal growth cones: Specific interactions mediated by filopodial insertion and induction of coated vesicles. Proc. Natl. Acad. Sci. USA. 81: 1849-1853.

Burkhardt, W., and V. Braitenberg (1976) Some peculiar synaptic complexes in the first visual ganglion of the fly, Musca domestica. Cell Tissue Res. 173: 287-308.

Coggshall, J. C. (1978) Neurons associated with the dorsal longitudinal flight muscles of Drosophila melanogaster. J. Comp. Neurol. 177: 707-720.

Costello, W. J. (1986) Formation of adult motor systems in Drosophila: Scanning electron microscope study. Soc. Neurosci. Abstr. 12: 930.

Costello, W. J., and M. R. Hummon (1985) Development of giant fiber pathway components in Drosophila: Possible involvement of coated vesicles. Soc. Neurosci. Abstr. 11:918.

Costello, W. J., and L. Salkoff (1983) Induced reduction in fiber number of indirect flight muscles in the Drosophila mutant shibire. Soc. Neurosci. Abstr. 9: 832.

Costello, W. J., and L. B. Salkoff (1986) Suppression of the membrane defect by divalent cations in the Drosophila mutant shibirc. J. Neurosci. $6: 3634-3639$.

Costello, W. J., and J. B. Thomas (1981) Development of thoracic muscles in muscle-specific mutant and normal Drosophila melanogaster. Soc. Neurosci. Abstr. 7: 543.

Costello, W. J., and R. J. Wyman (1986) Development of an indirect flight muscle in a muscle-specific mutant of Drosophila melanogaster. Dev. Biol. 118: 247-258.

Goodman, C. S., and K. G. Pearson (eds.) (1982) Neuronal development: Cellular approaches in invertebrates. Neurosci. Res. Program Bull. 20: 773-942.

Grigliatti, T. A., L. Hall, R. Rosenbluth, and D. T. Suzuki (1973) Temperature-sensitive mutations in Drosophila melanogaster. XIV. A selection of immobile adults. Mol. Gen. Genet. 120: 107-114.

Hirano, A., and W.-Y. Shin (1979) Unattached presynaptic terminals in a cerebellar neuroblastoma in the human. Neuropathol. Appl. Neurobiol. 5: 63-70.

Hummon, M. R., and W. J. Costello (1985) Altered development of the Drosophila giant fiber pathway in the temperature-sensitive $\mathrm{mu}$ - 
tant shibire: Control by timing of heat-pulse. Soc. Neurosci. Abstr. 11: 918 .

Hummon, M. R., and W. J. Costello (1986a) Induction of neuroma formation and specific target muscle deletion in the Drosophila ts mutant shibire. Soc. Neurosci. Abstr. 12: 929.

Hummon, M. R., and W. J. Costello (1986b) Giant fiber activation of flight muscles in Drosophila: Asynchrony of latency in fibers of the wing depressors [abstract]. Am. Zool. 26: 127A.

Kim, Y.-T., and C.-F. Wu (1986) Effects of altered membrane recycling process on neuronal development: Analysis of cultured neurons of the Drosophila mutant, shibirest. Soc. Neurosci. Abstr. 12: 1212.

Kim, Y.-T., and C.-F. Wu (1987) Reversible blockage of neurite development and growth cone formation in neuronal cultures of a temperature-sensitive mutant of Drosophila. J. Neurosci. 7: 3245-3255.

King, D. G., and R. J. Wyman (1980) Anatomy of the giant fibre pathway in Drosophila. I. Thoracic components of the pathway. $\mathbf{J}$. Neurocytol. 9: 753-770.

King, D. G., N. Kammlade, and J. Murphy (1982) A simple device to help re-embed thick plastic sections. Stain Technol. 57: 307-310.

Koenig, J. H., K. Saito, and K. Ikeda (1983) Reversible control of synaptic transmission in a single gene mutant of Drosophila melanogaster. J. Cell Biol. 96: 1517-1522.
Kosaka, T., and K. Ikeda (1983a) Possible temperature-dependent blockage of synaptic vesicle recycling induced by a single gene mutation in Drosophila. J. Neurobiol. 14: 207-225.

Kosaka, T., and K. Ikeda (1983b) Reversible blockage of membrane retrieval and endocytosis in the garland cell of the temperature-sensitive mutant of Drosophila melanogaster, shibire. J. Cell Biol. 97. 499-507.

Poodry, C. A., and L. Edgar (1979) Reversible alterations in the neuromuscular junctions of Drosophila melanogaster bearing a temperature-sensitive mutation, shibire. J. Cell Biol. 81: 520-527.

Poodry, C. A., L. Hall, and D. T. Suzuki (1973) Developmental properties of shibiress: A pleiotropic mutation affecting larval and adult locomotion and development. Dev. Biol. 32: 373-386.

Salkoff, L., and L. Kelly (1978) Temperature-induced seizure and frequency-dependent neuromuscular block in a $t s$ mutant of Drosophila. Nature 273: 156-158.

Sokal, R. R., and F. J. Rohlf (1969) Biometry. Freeman, San Francisco. Tanouye, M. A., and R. J. Wyman (1980) Motor outputs of giant nerve fiber in Drosophila. J. Neurophysiol. 44: 405-421.

Thomas, J. B., and R. J. Wyman (1984) Mutations altering synaptic connectivity between identified neurons in Drosophila. J. Neurosci. 4: $530-538$. 